\title{
Supertwistor realisations of AdS superspaces
}

\author{
Sergei M. Kuzenko ${ }^{1, a}$, Gabriele Tartaglino-Mazzucchelli ${ }^{2, b}$ \\ ${ }^{1}$ Department of Physics M013, The University of Western Australia, 35 Stirling Highway, Perth, WA 6009, Australia \\ ${ }^{2}$ School of Mathematics and Physics, University of Queensland, St Lucia, Brisbane, QLD 4072, Australia
}

Received: 1 November 2021 / Accepted: 27 January 2022 / Published online: 16 February 2022

(C) The Author(s) 2022

\begin{abstract}
We propose supertwistor realisations of $(p, q)$ anti-de Sitter (AdS) superspaces in three dimensions and $\mathcal{N}$-extended AdS superspaces in four dimensions. For each superspace, we identify a two-point function that is invariant under the corresponding isometry supergroup. This twopoint function is a supersymmetric extension (of a function) of the geodesic distance. We also describe a bi-supertwistor formulation for $\mathcal{N}$-extended AdS superspace in four dimensions and harmonic/projective extensions of $(p, q)$ AdS superspaces in three dimensions.
\end{abstract}

\section{Contents}

1 Introduction ................ 1

$2(p, q)$ AdS superspace in three dimensions . . . . 2

2.1 Algebraic background . . . . . . . . . . . 2

2.2 Supertwistor realisation of $(p, q)$ AdS superspace 3

$2.3 G$-invariant two-point function on $\operatorname{AdS}^{(3 \mid p, q)}$. 4

$3 \mathcal{N}$-extended AdS superspace in four dimensions . . 4

3.1 Algebraic background . . . . . . . . . . . . 4

3.2 Supertwistor realisation of $\operatorname{AdS}^{4 \mid 4 \mathcal{N}} \ldots \ldots .4$

3.3 Anti-de Sitter space . . . . . . . . . . . . . . 5

$3.4 \operatorname{OSp}(\mathcal{N} \mid 4 ; \mathbb{R})$-invariant two-point function on $\operatorname{AdS}^{4 \mid 4 \mathcal{N}} \ldots \ldots \ldots \ldots \ldots \ldots$

3.5 Poincaré coordinate patch in $\operatorname{AdS}^{4 \mid 4 \mathcal{N}} \ldots \ldots .5$

4 Bi-supertwistor construction for $\operatorname{AdS}^{4 / 4 \mathcal{N}} \ldots \ldots 6$

5 Harmonic/projective AdS superspaces . . . . . . . . 7

6 Conclusion . . . . . . . . . . . . . 7

A Compactified $(p, q)$ Minkowski superspace in two

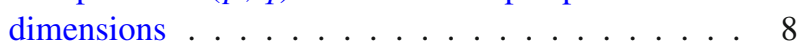

References................. 8

a e-mail: sergei.kuzenko@uwa.edu.au

b e-mail: g.tartaglino-mazzucchelli@uq.edu.au (corresponding author)

\section{Introduction}

Propagators in maximally symmetric spacetimes (see, e.g., [1-6] and references therein) make use of a unique two-point function which is invariant under the corresponding isometry group. Such a two-point function is readily constructed if one makes use of the well-known embedding formalisms for de Sitter and anti-de Sitter spaces. Off-shell supersymmetric field theories in $\mathrm{AdS}_{d}$ are naturally formulated in appropriate AdS superspaces for $d \leq 5$. In order to develop quantum supergraph techniques in such a superspace, it is useful to work with an embedding formalism. In this letter we propose supertwistor formulations for the following superspace types: (i) $(p, q)$ anti-de Sitter (AdS) superspace in three dimensions; and (ii) $\mathcal{N}$-extended AdS superspace in four dimensions.

Since the work by Ferber [7], supertwistors have found numerous applications in theoretical and mathematical physics. In particular, supertwistor realisations of compactified $\mathcal{N}$-extended Minkowski superspaces have been developed in four [8-10] and three [11,12] dimensions and their harmonic/projective extensions [11-19]. ${ }^{1}$ Recently, supertwistor formulations for conformal supergravity theories in diverse dimensions have been proposed [21,22]. Unlike in Minkowski space, not much is known about supertwistor realisations of AdS superspaces in diverse dimensions, to the best of our knowledge, although (super)twistor descriptions of (super)particles in AdS have been studied in the literature [23-32]. Our goal in this paper is to fill the gap. Of course, for theories in AdS it is always possible to use the standard coset space formalism, see, e.g., the famous Metsaev-Tseytlin construction of the type IIB superstring action in $\mathrm{AdS}_{5} \times \mathrm{S}^{5}$ [33]. However, manifest symmetry is one of the main virtues of (super)twistor techniques.

\footnotetext{
${ }^{1}$ Similar ideas were applied in Ref. [20] to develop supertwistor realisations of the $2 n$-extended supersphere $S^{3 \mid 4 n}$, with $n=1,2, \ldots$, as a homogeneous space of the three-dimensional Euclidean superconformal group $\operatorname{OSp}(2 n \mid 2,2)$.
} 
This paper is organised as follows. In Sect. 2 we present the supertwistor realisations of $(p, q)$ AdS superspace in three dimensions. Section 3 is devoted to the four-dimensional $\mathcal{N}$ extended case which is then extended to a bi-supertwistor construction in Sect. 4. Section 5 is devoted to supertwistor constructions of harmonic/projective AdS superspaces in three dimensions, while Sect. 6 contains concluding comments for our paper. In the Appendix we describe a supertwistor realisation of two-dimensional compactified Minkowski superspace $\overline{\mathrm{M}}^{(2 \mid p, q)}$.

\section{$2(p, q)$ AdS superspace in three dimensions}

The $(p, q)$ AdS superspaces in three dimensions (3D) were introduced in [34] as backgrounds of the off-shell 3D $\mathcal{N}$ extended conformal supergravity [35,36] with covariantly constant and Lorentz invariant torsion. In this paper we will restrict our attention to the conformally flat $(p, q)$ AdS superspaces $^{2}$

$\operatorname{AdS}^{(3 \mid p, q)}=\frac{\operatorname{OSp}(p \mid 2 ; \mathbb{R}) \times \operatorname{OSp}(q \mid 2 ; \mathbb{R})}{\operatorname{SL}(2, \mathbb{R}) \times \operatorname{SO}(p) \times \operatorname{SO}(q)}$,

which may be viewed as maximally supersymmetric solutions of $(p, q)$ AdS supergravity theories [37] (even though these theories are intrinsically formulated in components without auxiliary fields and can be recast in superspace only on the mass shell). ${ }^{3}$ The superspaces (2.1) with $p+q \leq 4$ naturally originate as maximally supersymmetric solution of various off-shell AdS supergravity theories. In particular, $\operatorname{AdS}{ }^{(3 \mid 1,0)}$ corresponds to $\mathcal{N}=1$ AdS supergravity [39]. The superspaces $\operatorname{AdS}^{(3 \mid 1,1)}$ and $\operatorname{AdS}{ }^{(3 \mid 2,0)}$ correspond to the off-shell formulations for $\mathcal{N}=2 \mathrm{AdS}$ supergravity given in $[36,40]$.

As demonstrated in [34], the isometry group of $\operatorname{AdS}^{(3 \mid p, q)}$ is

$G=\operatorname{OSp}(p \mid 2 ; \mathbb{R}) \times \operatorname{OSp}(q \mid 2 ; \mathbb{R}) \equiv G_{\mathrm{L}} \times G_{\mathrm{R}}$

The same supergroup is also the superconformal group of compactified Minkowski superspace in two dimensions, $\overline{\mathbb{M}}^{(2 \mid p, q)}$, with its bosonic body being $\overline{\mathbb{M}}^{2}=S^{1} \times S^{1}$, the compactified two-dimensional Minkowski space. ${ }^{4}$ Our embedding formalism for $\operatorname{AdS}^{(3 \mid p, q)}$ is constructed in terms of $2 \mathrm{D}$ supertwistors.

\footnotetext{
${ }^{2}$ In the case $(p, q)=(\mathcal{N}, 0)$ there also exist non-conformally flat AdS superspaces if $\mathcal{N} \geq 4$ [34]. They will be discussed elsewhere.

3 The coset spaces (2.1) were briefly discussed in [38].

${ }^{4}$ The supertwistor realisation of $\overline{\mathbb{M}}^{(2 \mid p, q)}$ is given in Appendix A.
}

\subsection{Algebraic background}

We introduce two types of pure supertwistors, (i) a left supertwistor

$T_{\mathrm{L}}=\left(T_{\bar{A}}\right)=\left(\begin{array}{c}T_{\bar{\alpha}} \\ T_{\bar{I}}\end{array}\right), \quad \bar{\alpha}=1,2, \quad \bar{I}=1, \ldots, p ;$

and (ii) a right supertwistor

$T_{\mathrm{R}}=\left(T_{\underline{A}}\right)=\left(\begin{array}{l}T_{\underline{\alpha}} \\ T_{\underline{I}}\end{array}\right), \quad \underline{\alpha}=1,2, \quad \underline{I}=1, \ldots, q$.

In the case of even left supertwistors, $T_{\bar{\alpha}}$ is bosonic and $T_{\bar{I}}$ is fermionic. In the case of odd left supertwistors, $T_{\bar{\alpha}}$ is fermionic while $T^{\bar{I}}$ is bosonic. The even and odd left supertwistors are called pure. We introduce the parity function $\varepsilon(T)$ defined as: $\varepsilon(T)=0$ if $T$ is even, and $\varepsilon(T)=1$ if $T$ is odd. Then the components $T_{\bar{A}}$ of a pure left supertwistor have the following Grassmann parities

$\varepsilon\left(T_{\bar{A}}\right)=\varepsilon(T)+\varepsilon_{\bar{A}}(\bmod 2)$,

where we have defined

$\varepsilon_{\bar{A}}=\left\{\begin{array}{ll}0 & \bar{A}=\bar{\alpha} \\ 1 & \bar{A}=\bar{I}\end{array}\right.$.

Analogous definitions are introduced for the right supertwistors.

A pure left supertwistor is said to be real if its components obey the reality condition

$\left(T_{\bar{A}}\right)^{*}=(-1)^{\varepsilon(T) \varepsilon_{\bar{A}}+\varepsilon_{\bar{A}}} T_{\bar{A}}$.

Real right supertwistors are similarly defined. The space of complex (real) even left supertwistors is naturally identified with $\mathbb{C}^{2 \mid p}\left(\mathbb{R}^{2 \mid p}\right)$, while the space of complex (real) odd left supertwistors may be identified with $\mathbb{C}^{p \mid 2}\left(\mathbb{R}^{p \mid 2}\right)$.

We introduce graded antisymmetric supermatrices $\mathbb{J}_{\mathrm{L}}$ and $\mathbb{J}_{\mathrm{R}}$ defined by

$$
\begin{aligned}
& \mathbb{J}_{\mathrm{L}}=\left(\mathbb{J}^{\bar{A} \bar{B}}\right)=\left(\begin{array}{c||c}
\varepsilon_{\mathrm{L}} & 0 \\
\hline \hline 0 & \mathrm{i} \mathbb{1}_{p}
\end{array}\right), \\
& \varepsilon_{\mathrm{L}}=\left(\varepsilon^{\bar{\alpha} \bar{\beta}}\right)=\left(\begin{array}{cc}
0 & 1 \\
-1 & 0
\end{array}\right),
\end{aligned}
$$

and similarly for $\mathbb{J}_{\mathrm{R}}$. Here $\mathbb{1}_{p}$ denotes the unit $p \times p$ matrix. Associated with $\rrbracket_{L}$ and $\rrbracket_{R}$ are graded symplectic inner products on the spaces of pure left and right supertwistors, respectively. For arbitrary pure left supertwistors $T$ and $S$, their inner product is

$\langle T \mid S\rangle_{\mathbb{J}_{\mathrm{L}}}:=T^{\mathrm{sT}} \mathbb{ل}_{\mathrm{L}} S$,

where the row vector $T^{\mathrm{sT}}$ is defined by

$T^{\mathrm{sT}}:=\left(T_{\bar{\alpha}},-(-1)^{\varepsilon(T)} T_{\bar{I}}\right)=\left(T_{\bar{A}}(-1)^{\varepsilon(T) \varepsilon_{\bar{A}}+\varepsilon_{\bar{A}}}\right)$ 
and is called the super-transpose of $T$. The above inner product is characterised by the symmetry property

$\left\langle T_{1} \mid T_{2}\right\rangle_{\mathbb{J}_{\mathrm{L}}}=-(-1)^{\varepsilon\left(T_{1}\right) \varepsilon\left(T_{2}\right)}\left\langle T_{2} \mid T_{1}\right\rangle_{\mathbb{J}_{\mathrm{L}}}$.

If $T_{1}$ and $T_{2}$ are real supertwistors, their inner product obeys the reality relation

$\left(\left\langle T_{1} \mid T_{2}\right\rangle_{\mathbb{J}_{\mathrm{L}}}\right)^{*}=-\left\langle T_{2} \mid T_{1}\right\rangle_{\mathbb{J}_{\mathrm{L}}}$

We recall that the supergroup $\operatorname{OSp}(p \mid 2 ; \mathbb{C})$ consists of those even $(2 \mid p) \times(2 \mid p)$ supermatrices

$g=\left(g_{\bar{A}}{ }^{\bar{B}}\right), \quad \varepsilon\left(g_{\bar{A}}{ }^{\bar{B}}\right)=\varepsilon_{\bar{A}}+\varepsilon_{\bar{B}}$,

which preserve the inner product (2.8) under the action

$T_{\mathrm{L}}=\left(T_{\bar{A}}\right) \rightarrow g \cdot T_{\mathrm{L}}=\left(g_{\bar{A}}{ }^{\bar{B}} T_{\bar{B}}\right)$.

Such a transformation maps the space of even (odd) supertwistors onto itself. The condition of invariance of the inner product (2.8) under (2.13) is

$g^{\mathrm{sT}} \mathbb{J}_{\mathrm{L}} g=\mathbb{J}_{\mathrm{L}}$,

where $g^{\mathrm{sT}}$ is the super-transpose of $g$ defined by

$\left(g^{\mathrm{sT}}\right)^{\bar{A}} \bar{B}:=(-1)^{\varepsilon} \bar{A}^{\varepsilon} \bar{B}^{-}+\varepsilon_{\bar{B}} g_{\bar{B}} \bar{A}$.

The subgroup $G_{\mathrm{L}} \equiv \mathrm{OSp}(p \mid 2 ; \mathbb{R}) \subset \mathrm{OSp}(p \mid 2 ; \mathbb{C})$ consists of those transformations which preserve the reality condition (2.6), which means

$\left(g_{\bar{A}} \bar{B}\right)^{*}=(-1)^{\varepsilon} \bar{A}^{\varepsilon} \bar{B}^{+\varepsilon} \bar{A} g_{\bar{A}} \bar{B} \quad \Longleftrightarrow g^{\dagger}=g^{\mathrm{sT}}$.

In conjunction with (2.14), this reality condition is equivalent to

$g^{\dagger} \mathbb{J}_{\mathrm{L}} g=\mathbb{J}_{\mathrm{L}}$.

Analogous definitions are introduced for the right supergroup $G_{\mathrm{R}} \equiv \operatorname{OSp}(q \mid 2 ; \mathbb{R}) \subset \operatorname{OSp}(q \mid 2 ; \mathbb{C})$.

\subsection{Supertwistor realisation of $(p, q)$ AdS superspace}

In order to obtain a supertwistor realisation of $(p, q) \mathrm{AdS}$ superspace, we introduce a space $\mathfrak{L}_{(p, q)}$. By definition, it consists of all pairs $\left(\mathcal{P}_{\mathrm{L}}, \mathcal{P}_{\mathrm{R}}\right)$, where

$\mathcal{P}_{\mathrm{L}}=\left(X_{\bar{A}}{ }^{\mu}\right), \quad \mu=1,2$

is a left real even two-plane, and

$\mathcal{P}_{\mathrm{R}}=\left(Y_{\underline{A}}^{\mu}\right), \quad \mu=1,2$

is a right real even two-plane, with the additional property

$\mathcal{P}_{\mathrm{L}}^{\mathrm{sT}} \mathbb{J}_{\mathrm{L}} \mathcal{P}_{\mathrm{L}}=\mathcal{P}_{\mathrm{R}}^{\mathrm{sT}} \mathbb{J}_{\mathrm{R}} \mathcal{P}_{\mathrm{R}}$

A few comments are in order. The statement that $\mathcal{P}_{\mathrm{L}}$ is even real, means that the two supertwistors $X_{\mathrm{L}}^{\mu}$ are even and real.
The property of $\mathcal{P}_{\mathrm{L}}$ being a two-plane means that ${ }^{5}$

$\operatorname{det}\left(X_{\bar{\alpha}}^{\mu}\right) \neq 0$

Similar statements hold for the right planes. In the space $\mathfrak{L}_{(p, q)}$ we introduce the following equivalence relation

$\left(\mathcal{P}_{\mathrm{L}}, \mathcal{P}_{\mathrm{R}}\right) \sim\left(\mathcal{P}_{\mathrm{L}} M, \mathcal{P}_{\mathrm{R}} M\right), \quad M \in \mathrm{GL}(2, \mathbb{R})$

The supergroup (2.2) acts on $\mathfrak{L}_{(p, q)}$ by the rule

$$
\begin{aligned}
& \left(g_{\mathrm{L}}, g_{\mathrm{R}}\right)\left(\mathcal{P}_{\mathrm{L}}, \mathcal{P}_{\mathrm{R}}\right):=\left(g_{\mathrm{L}} \mathcal{P}_{\mathrm{L}}, g_{\mathrm{R}} \mathcal{P}_{\mathrm{R}}\right) \\
& \quad\left(g_{\mathrm{L}}, g_{\mathrm{R}}\right) \in \operatorname{OSp}(p \mid 2 ; \mathbb{R}) \times \operatorname{OSp}(q \mid 2 ; \mathbb{R}) .
\end{aligned}
$$

This action is naturally extended to the quotient space $\mathfrak{L}_{(p, q)} / \sim$. The latter proves to be a homogeneous space of $\operatorname{OSp}(p \mid 2 ; \mathbb{R}) \times \operatorname{OSp}(q \mid 2 ; \mathbb{R})$. It turns out that

$\operatorname{AdS}^{(3 \mid p, q)}=\mathfrak{L}_{(p, q)} / \sim$

The equivalence relation (2.20) allows us to choose a gauge

$\mathcal{P}_{\mathrm{R}}=\left(Y_{\underline{A}^{\mu}}^{\mu}\right)=\left(\begin{array}{c}\delta_{\underline{\alpha}}^{\mu} \\ \mathrm{i} \bar{\theta}_{\underline{I}}^{\mu}\end{array}\right), \quad \mathcal{P}_{\mathrm{L}}=\left(X_{\bar{A}}^{\mu}\right)=\left(\begin{array}{c}x_{\bar{\alpha}}^{\mu} \\ \mathrm{i} \theta_{\bar{I}}^{\mu}\end{array}\right)$.

Then the condition (2.18) turns into

$x^{\mathrm{T}} \varepsilon x=\varepsilon-\mathrm{i}\left(\theta_{\mathrm{L}}^{\mathrm{T}} \theta_{\mathrm{L}}-\theta_{\mathrm{R}}^{\mathrm{T}} \theta_{\mathrm{R}}\right)$.

This equation provides the embedding of $\operatorname{AdS}^{(3 \mid p, q)}$ into $\mathbb{R}^{2,2 \mid 2 p+2 q}$. In the non-supersymmetric case, $p=q=0$, $(2.23 b)$ is equivalent to

$x \in \operatorname{Sp}(2, \mathbb{R}) \cong \operatorname{SL}(2, \mathbb{R})$,

which is the standard realisation of $\mathrm{AdS}_{3}$.

Instead of using the gauge (2.23a), one can choose the alternative gauge condition

$$
\mathcal{P}_{\mathrm{L}}=\left(X_{\bar{A}}{ }^{\mu}\right)=\left(\begin{array}{c}
\delta_{\bar{\alpha}}^{\mu} \\
\mathrm{i} \vartheta_{\bar{I}}^{\mu}
\end{array}\right), \quad \mathcal{P}_{\mathrm{R}}=\left(Y_{\underline{A}^{\mu}}^{\mu}\right)=\left(\begin{array}{c}
y_{\underline{\alpha}^{\mu}}^{\mu} \\
\mathrm{i} \vartheta_{\underline{I}}^{\mu}
\end{array}\right)
$$

Then the condition (2.18) turns into

$y^{\mathrm{T}} \varepsilon y=\varepsilon-\mathrm{i}\left(\vartheta_{\mathrm{R}}^{\mathrm{T}} \vartheta_{\mathrm{R}}-\vartheta_{\mathrm{L}}^{\mathrm{T}} \vartheta_{\mathrm{L}}\right)$

\footnotetext{
$\overline{5}$ More precisely, the body of the matrix $\left(X_{\bar{\alpha}}{ }^{\mu}\right)$ must be a nonsingular matrix. See [41] for the necessary information about infinite dimensional Grassmann algebra $\Lambda_{\infty}$ and supermatrices.
} 
$2.3 G$-invariant two-point function on $\operatorname{AdS}^{(3 \mid p, q)}$

Let $Z=\left(\mathcal{P}_{\mathrm{L}}, \mathcal{P}_{\mathrm{R}}\right)$ and $\widetilde{Z}=\left(\widetilde{\mathcal{P}}_{\mathrm{L}}, \widetilde{\mathcal{P}}_{\mathrm{R}}\right)$ be two points of $\mathfrak{L}_{(p, q)}$. We introduce the following two-point function ${ }^{6}$

$\omega(Z, \widetilde{Z})=\frac{1}{2} \operatorname{tr}\left\{\widetilde{\mathcal{P}}_{\mathrm{L}}^{\mathrm{sT}} \mathbb{J}_{\mathrm{L}} \mathcal{P}_{\mathrm{L}}\left(\widetilde{\mathcal{P}}_{\mathrm{R}}^{\mathrm{sT}} \mathbb{J}_{\mathrm{R}} \mathcal{P}_{\mathrm{R}}\right)^{-1}\right\}$.

By construction, it is invariant under the group action (2.21). The two-point function is also well defined on the quotient space (2.22). Indeed, given two sets of equivalent points

$\left(\mathcal{P}_{\mathrm{L}}, \mathcal{P}_{\mathrm{R}}\right) \sim\left(\mathcal{P}_{\mathrm{L}} M, \mathcal{P}_{\mathrm{R}} M\right), \quad\left(\widetilde{\mathcal{P}}_{\mathrm{L}}, \widetilde{\mathcal{P}}_{\mathrm{R}}\right) \sim\left(\widetilde{\mathcal{P}}_{\mathrm{L}} \tilde{M}, \widetilde{\mathcal{P}}_{\mathrm{R}} \tilde{M}\right)$,

with $M, \tilde{M} \in \mathrm{GL}(2, \mathbb{R})$, we have

$$
\begin{aligned}
& \widetilde{\mathcal{P}}_{\mathrm{L}}^{\mathrm{sT}} \mathbb{J}_{\mathrm{L}} \mathcal{P}_{\mathrm{L}} \sim \widetilde{M}^{\mathrm{T}} \widetilde{\mathcal{P}}_{\mathrm{L}}^{\mathrm{sT}} \mathbb{J}_{\mathrm{L}} \mathcal{P}_{\mathrm{L}} M, \\
& \widetilde{\mathcal{P}}_{\mathrm{R}}^{\mathrm{sT}} \mathbb{J}_{\mathrm{R}} \mathcal{P}_{\mathrm{R}} \sim \tilde{M}^{\mathrm{T}} \widetilde{\mathcal{P}}_{\mathrm{R}}^{\mathrm{sT}} \mathbb{J}_{\mathrm{R}} \mathcal{P}_{\mathrm{R}} M,
\end{aligned}
$$

and therefore the two-point function (2.26) does not change. It is instructive to evaluate (2.26) in the non-supersymmetric case, $p=q=0$. Assuming the gauge condition (2.23a), we then have

$$
\begin{gathered}
x=\left(\begin{array}{ll}
x^{0}+x^{1} & x^{2}+x^{3} \\
x^{2}-x^{3} & x^{0}-x^{1}
\end{array}\right), \\
\left(x^{0}\right)^{2}+\left(x^{3}\right)^{2}-\left(x^{1}\right)^{2}-\left(x^{2}\right)^{2}=1,
\end{gathered}
$$

and therefore

$w(x, \tilde{x})=\tilde{x}^{0} x^{0}+\tilde{x}^{3} x^{3}-\tilde{x}^{1} x^{1}-\tilde{x}^{2} x^{2}$.

\section{$3 \mathcal{N}$-extended AdS superspace in four dimensions}

The supergroup $\operatorname{OSp}(\mathcal{N} \mid 4 ; \mathbb{R})$ is the isometry group of fourdimensional $\mathcal{N}$-extended AdS superspace

$\operatorname{AdS}^{4 \mid 4 \mathcal{N}}=\frac{\mathrm{OSp}(\mathcal{N} \mid 4 ; \mathbb{R})}{\operatorname{SO}(3,1) \times \operatorname{SO}(\mathcal{N})}$.

Here we describe a supertwistor realisation of this superspace. Our embedding formalism for $\operatorname{AdS}^{4 \mid 4 \mathcal{N}}$ is constructed in terms of 3D supertwistors.

It should be pointed out that $\mathrm{AdS}^{4 / 4}$ was introduced in [4244]. It is a maximally supersymmetric solution of $\mathcal{N}=1$ supergravity with a cosmological term, see $[39,41]$ for a review. The description of $\operatorname{AdS}^{4 \mid 8}$ as a maximally supersymmetric solution of $\mathcal{N}=2$ supergravity with a cosmological term was given in [45-47]. The conformal flatness of $\mathrm{AdS}^{4 / 4}$ was established by Ivanov and Sorin [44] and then reviewed in textbooks $[39,41]$. The superconformal flatness of $\operatorname{AdS}^{4 \mid 4 \mathcal{N}}$ was demonstrated in [38]. Ref. [48] described alternative conformally flat realisations for $\operatorname{AdS}^{4 \mid 4}$

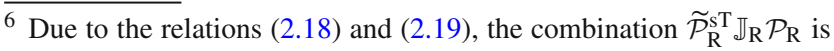
nonsingular. and $\operatorname{AdS}^{4 \mid 8}$ which are based on the use of Poincaré coordinates.

\subsection{Algebraic background}

A supertwistor is a column vector

$$
\begin{aligned}
& T=\left(T_{A}\right)=\left(\frac{T_{\hat{\alpha}}}{\overline{T_{i}}}\right), \quad\left(T_{\hat{\alpha}}\right)=\left(\begin{array}{c}
f_{\alpha} \\
g^{\beta}
\end{array}\right), \\
& \alpha, \beta=1,2 \quad i=1, \ldots, \mathcal{N} .
\end{aligned}
$$

Pure supertwistors are defined similarly to Sect. 2. Specifically the components $T_{A}$ of a pure supertwistor have the following Grassmann parities

$\varepsilon\left(T_{A}\right)=\varepsilon(T)+\varepsilon_{A} \quad(\bmod 2)$,

where we have defined

$\varepsilon_{A}=\left\{\begin{array}{cc}0 & A=\hat{\alpha} \\ 1 & A=i\end{array}\right.$.

We choose the graded antisymmetric supermatrix

$$
\begin{aligned}
& \mathbb{J}=\left(\mathbb{J}^{A B}\right)=\left(\begin{array}{c||c}
J & 0 \\
\hline \overline{0} & \mathrm{i} \mathbb{1}_{\mathcal{N}}
\end{array}\right), \\
& J=\left(J^{\hat{\alpha} \hat{\beta}}\right)=\left(\begin{array}{cc}
0 & \mathbb{1}_{2} \\
-\mathbb{1}_{2} & 0
\end{array}\right),
\end{aligned}
$$

which allows us to define a graded symplectic inner product on the space of pure supertwistors by the rule: for arbitrary pure supertwistors $T$ and $S$, the inner product is

$$
\langle T \mid S\rangle_{\mathbb{J}}:=T^{\mathrm{sT}} \mathbb{J} S
$$

\subsection{Supertwistor realisation of $\operatorname{AdS} S^{4 \mid 4 \mathcal{N}}$}

We denote by $\mathfrak{E}_{\mathcal{N}}$ the space of all real even supertwistors. Next we introduce a complex frame in $\mathfrak{E}_{\mathcal{N}}$

$$
\begin{aligned}
& T^{\hat{\mu}}=\left(T^{\mu}, \bar{T}^{\dot{\mu}}\right), \quad T^{\mu}=\left(T_{A}{ }^{\mu}\right), \quad \bar{T}^{\dot{\mu}}=\left(\bar{T}_{A} \dot{\mu}\right) . \\
& \mu, \dot{\mu}=1,2 .
\end{aligned}
$$

Here the supertwistor $\bar{T}^{\dot{\mu}}$ is the complex conjugate of $T^{\mu}$. We require the elements of the frame to obey the conditions:

$\varepsilon_{\mu \nu}\left\langle T^{\mu} \mid T^{v}\right\rangle_{\mathbb{J}} \neq 0$

$$
\left\langle T^{\mu} \mid \bar{T}^{\dot{v}}\right\rangle_{\mathbb{J}}=0 .
$$

We denote $\mathfrak{F}_{\mathcal{N}}$ the space of all complex frames (3.6).

It is not difficult to construct explicit examples of complex frames (3.6). Let $U^{\mu}$ and $V^{\mu}$ be real even supertwistors with the properties

$$
\begin{aligned}
& \left\langle U^{\mu} \mid U^{\nu}\right\rangle_{\mathbb{J}}=\left\langle V^{\mu} \mid V^{\nu}\right\rangle_{\mathbb{J}}=0, \\
& \left\langle U^{\mu} \mid V^{\nu}\right\rangle_{\mathbb{J}}=-\left\langle V^{\mu} \mid U^{\nu}\right\rangle_{\mathbb{J}}=\delta^{\mu \nu} .
\end{aligned}
$$


Such supertwistors originate as even vector-columns of an arbitrary group element $g \in \operatorname{OSp}(\mathcal{N} \mid 4 ; \mathbb{R})$. Then we define the complex even supertwistors

$T^{\mu}:=U^{\mu}+\mathrm{i} \varepsilon^{\mu \sigma} V^{\sigma}, \quad \bar{T}^{\dot{\mu}}:=U^{\mu}-\mathrm{i} \varepsilon^{\mu \sigma} V^{\sigma}$,

for which the properties (3.6) hold.

In the space of frames $\mathfrak{F}_{\mathcal{N}}$, we introduce the following equivalence relation

$T^{\mu} \sim T^{\nu} R_{\nu}{ }^{\mu}, \quad R \in \mathrm{GL}(2, \mathbb{C})$.

The supergroup $\operatorname{OSp}(\mathcal{N} \mid 4 ; \mathbb{R})$ acts on $\mathfrak{F}_{\mathcal{N}}$ by the rule

$g\left(T^{\mu}, \bar{T}^{\dot{\mu}}\right)=\left(g T^{\mu}, g \bar{T}^{\dot{\mu}}\right), \quad g \in \operatorname{OSp}(\mathcal{N} \mid 4 ; \mathbb{R})$.

This action is naturally extended to the quotient space $\mathfrak{F}_{\mathcal{N}} / \sim$. The latter proves to be a homogeneous space of $\operatorname{OSp}(\mathcal{N} \mid 4 ; \mathbb{R})$. It turns out that

$\operatorname{AdS}^{4 \mid 4 \mathcal{N}}=\mathfrak{F}_{\mathcal{N}} / \sim$.

\subsection{Anti-de Sitter space}

In order to prove (3.11), it suffices to consider the nonsupersymmetric case, $\mathcal{N}=0$. Then we have

$$
\begin{aligned}
& T_{\hat{\alpha}}^{\hat{\mu}} T_{\hat{\beta}}^{\hat{v}} T_{\hat{\gamma}}^{\hat{\sigma}} T_{\hat{\delta}}^{\hat{\rho}} \varepsilon_{\hat{\mu} \hat{v} \hat{\sigma} \hat{\rho}}=\Delta \varepsilon_{\hat{\alpha} \hat{\beta} \hat{\gamma} \hat{\delta}} \\
& =-\Delta\left(J_{\hat{\alpha} \hat{\beta}} J_{\hat{\gamma} \hat{\delta}}+J_{\hat{\alpha} \hat{\gamma}} J_{\hat{\delta} \hat{\beta}}+J_{\hat{\alpha} \hat{\delta}} J_{\hat{\beta} \hat{\gamma}}\right),
\end{aligned}
$$

for some $\Delta \neq 0$. We know that

$\left\langle T^{\mu} \mid T^{\nu}\right\rangle_{\mathbb{J}}=\kappa \varepsilon^{\mu \nu}, \quad\left\langle\bar{T}^{\dot{\mu}} \mid \bar{T}^{\dot{v}}\right\rangle_{\mathbb{J}}=\bar{\kappa} \varepsilon^{\dot{\mu} \dot{v}}$,

for some complex parameter $\kappa \neq 0$. Making use of (3.6a), (3.12) and (3.13), we deduce that

$\bar{\kappa} T_{\hat{\alpha}}^{\mu} T_{\hat{\beta} \mu}+\kappa \bar{T}_{\hat{\alpha}}^{\dot{\mu}} \bar{T}_{\hat{\beta} \dot{\mu}}=-\Delta J_{\hat{\alpha} \hat{\beta}}$.

It is useful to introduce the traceless part of the antisymmetric bi-twistor $T_{\hat{\alpha}}^{\mu} T_{\hat{\beta} \mu}$,

$T_{\langle\hat{\alpha}}^{\mu} T_{\hat{\beta}\rangle \mu}=T_{\hat{\alpha}}^{\mu} T_{\hat{\beta} \mu}-\frac{1}{2} J_{\hat{\alpha} \hat{\beta}} \kappa, \quad J^{\hat{\alpha} \hat{\beta}} T_{\langle\hat{\alpha}}^{\mu} T_{\hat{\beta}\rangle \mu}=0$.

Then the relation (3.14) is equivalent to the two identities:

$$
\begin{aligned}
\bar{\kappa} T_{\langle\hat{\alpha}}{ }^{\mu} T_{\hat{\beta}\rangle \mu}+\kappa \bar{T}_{\langle\hat{\alpha}} \dot{\mu} \bar{T}_{\hat{\beta}\rangle \dot{\mu}} & =0, \\
\Delta & =-\kappa \bar{\kappa} .
\end{aligned}
$$

Making use of the equivalence relation (3.9) allows us to choose a gauge

$\kappa=-\bar{\kappa}=\mathrm{i} \ell$,

for a fixed real parameter $\ell$. Then (3.16a) turns into the reality condition

$T_{\langle\hat{\alpha}}^{\mu} T_{\hat{\beta}\rangle \mu}=\bar{T}_{\langle\hat{\alpha}}{ }^{\dot{\mu}} \bar{T}_{\hat{\beta}\rangle \dot{\mu}}$.
Associated with $T_{\langle\hat{\alpha}}^{\mu} T_{\hat{\beta}\rangle \mu}$ is the real 5-vector

$X_{\hat{a}}:=\frac{1}{2}\left(J \Gamma_{\hat{a}}\right)^{\hat{\alpha} \hat{\beta}} T_{\langle\hat{\alpha}}^{\mu} T_{\hat{\beta}\rangle \mu}=\frac{1}{2}\left(J \Gamma_{\hat{a}}\right)^{\hat{\alpha} \hat{\beta}} T_{\hat{\alpha}}{ }^{\mu} T_{\hat{\beta} \mu}$.

Here $\Gamma_{\hat{a}}$ are real $4 \times 4$ matrices which obey the anticommutation relations

$$
\begin{aligned}
& \left\{\Gamma_{\hat{a}}, \Gamma_{\hat{b}}\right\}=2 \eta_{\hat{a} \hat{b}} \mathbb{1}_{4}, \quad \eta_{\hat{a} \hat{b}}=\operatorname{diag}(-+++-), \\
& \hat{a}=0,1,2,3,4 \equiv a, 3,4 .
\end{aligned}
$$

These matrices constitute a Majorana representation of the gamma-matrices for pseudo-Euclidean space $\mathbb{R}^{3,2}$. The explicit realisation of $\Gamma_{\hat{a}}$ is given, e.g., in [12]. Making use of the completeness relation

$\left(J \Gamma^{\hat{a}}\right)^{\hat{\alpha} \hat{\beta}}\left(J \Gamma_{\hat{a}}\right)^{\hat{\gamma} \hat{\delta}}=-J^{\hat{\alpha} \hat{\beta}} J^{\hat{\gamma} \hat{\delta}}+2\left(J^{\hat{\alpha} \hat{\gamma}} J^{\hat{\beta} \hat{\delta}}-J^{\hat{\alpha} \hat{\delta}} J^{\hat{\beta} \hat{\gamma}}\right)$,

we obtain

$X^{\hat{a}} X_{\hat{a}}=-\ell^{2}$.

The above twistor description of $\mathrm{AdS}_{4}$ is equivalent to the bispinor formalism introduced in [49].

\section{4 $\operatorname{OSp}(\mathcal{N} \mid 4 ; \mathbb{R})$-invariant two-point function on $\operatorname{AdS} S^{4 \mid 4 \mathcal{N}}$}

Let $T^{\hat{\mu}}$ and $\widetilde{T}^{\hat{\mu}}$ be arbitrary points of $\mathfrak{F}_{\mathcal{N}}$. The following two-point function

$\omega(T, \widetilde{T}):=\frac{\left\langle\bar{T}^{\dot{\mu}} \mid \widetilde{T}^{\nu}\right\rangle_{\mathbb{J}}\left\langle\bar{T}_{\dot{\mu}} \mid \widetilde{T}_{\nu}\right\rangle_{\mathbb{J}}}{\left\langle\bar{T}^{\dot{\sigma}} \mid \bar{T}_{\dot{\sigma}}\right\rangle_{\mathbb{J}}\left\langle\widetilde{T}^{\rho} \mid \widetilde{T}_{\rho}\right\rangle_{\mathbb{J}}}$

is clearly $\operatorname{OSp}(\mathcal{N} \mid 4 ; \mathbb{R})$-invariant. It is also invariant under equivalence transformations

$T^{\mu} \rightarrow T^{v} R_{\nu}{ }^{\mu}, \quad \widetilde{T}^{\mu} \rightarrow \widetilde{T}^{v} \widetilde{R}_{\nu}{ }^{\mu}, \quad R, \widetilde{R} \in \mathrm{GL}(2, \mathbb{C})$,

and therefore the two-point function is defined on the quotient space (3.11).

In the non-supersymmetric case, $\mathcal{N}=0,(3.23)$ is simply related to the $\mathrm{AdS}_{4}$ two-point function $X^{\hat{a}} \widetilde{X}_{\hat{a}}$. In the gauge (3.17), we obtain

$X^{\hat{a}} \widetilde{X}_{\hat{a}}=-\ell^{2}+\left\langle\bar{T}^{\dot{\mu}} \mid \widetilde{T}^{v}\right\rangle_{\mathbb{J}}\left\langle\bar{T}_{\dot{\mu}} \mid \widetilde{T}_{\nu}\right\rangle_{\mathbb{J}}$.

\subsection{Poincaré coordinate patch in $\operatorname{AdS}^{4 \mid 4 \mathcal{N}}$}

Let us consider an open subset of $\operatorname{AdS}^{4 \mid 4 \mathcal{N}}$ such that the upper $2 \times 2$ block in

$T^{\mu}=\left(\frac{\frac{T_{\alpha}{ }^{\mu}}{T^{\beta \mu}}}{\overline{T_{I}{ }^{\mu}}}\right)$ 
is nonsingular. Then we can use the gauge freedom (3.9) to impose the condition (3.17) and choose $T_{\alpha}{ }^{\mu} \propto \delta_{\alpha}{ }^{\mu}$. Now, imposing the conditions (3.6c), (3.13) and (3.17), we obtain the general solution

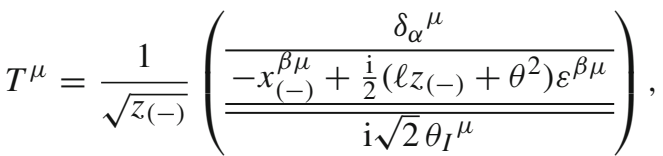

$$
\begin{aligned}
& \bar{T}^{\dot{\mu}}=\frac{1}{\sqrt{z_{(+)}}}\left(\frac{\frac{\delta_{\alpha} \dot{\mu}}{-x_{(+)}^{\beta \dot{\mu}}-\frac{\mathrm{i}}{2}\left(\ell_{(+)}-\bar{\theta}^{2}\right) \varepsilon^{\beta \dot{\mu}}}}{\mathrm{i} \sqrt{2} \bar{\theta}_{I}^{\dot{\mu}}}\right) \text {, }
\end{aligned}
$$

where we have denoted

$$
\begin{array}{r}
\left.x_{( \pm)}^{\alpha \beta}=x^{\alpha \beta} \pm \mathrm{i} \theta_{I}{ }^{(\alpha} \bar{\theta}_{I} \beta\right), \quad x^{\alpha \beta}=\left(\begin{array}{cc}
x^{0}-x^{2} & -x^{1} \\
-x^{1} & x^{0}+x^{2}
\end{array}\right), \\
(3.28 \mathrm{a}) \\
z_{( \pm)}=z \pm \frac{1}{2 \ell}(\theta-\bar{\theta})^{2}, \quad \theta^{2}=\theta_{I}{ }^{\alpha} \theta_{I \alpha}, \quad \bar{\theta}^{2}=\bar{\theta}_{I}{ }^{\alpha} \bar{\theta}_{I \alpha} .
\end{array}
$$

The real coordinates $z>0$ and $x^{a}=\left(x^{0}, x^{1}, x^{2}\right)$ parametrise $\mathrm{AdS}_{4}$ in the Poincaré patch. They are related to the embedding coordinates $X^{\hat{a}}$, eq. (3.22), as follows

$$
\begin{aligned}
X^{\hat{a}} & =\left(X^{a}, X^{3}, X^{4}\right) \\
& =\frac{1}{z}\left(x^{a}, \frac{1-x^{2}-(\ell z)^{2}}{2}, \frac{1+x^{2}+(\ell z)^{2}}{2}\right), \\
x^{2} & =x^{a} x_{a} .
\end{aligned}
$$

In the non-supersymmetric case, $\mathcal{N}=0$, the relations (3.27) reduce to those given in [49].

Using the Poincaré coordinates introduced, it is easy to show that the $\operatorname{OSp}(\mathcal{N} \mid 4 ; \mathbb{R})$-invariant two-point function (3.23) is not unique in the $\mathcal{N}>0$ case. In addition to (3.23), we can introduce the following two-point functions

$$
\begin{gathered}
\omega_{(-)}(T, \widetilde{T}):=\frac{\left\langle T^{\mu} \mid \widetilde{T}^{\nu}\right\rangle_{\mathbb{J}}\left\langle T_{\mu} \mid \widetilde{T}_{\nu}\right\rangle_{\mathbb{J}}}{\left\langle T^{\sigma} \mid T_{\sigma}\right\rangle_{\mathbb{J}}\left\langle\widetilde{T}^{\rho} \mid \widetilde{T}_{\rho}\right\rangle_{\mathbb{J}}}, \\
\omega_{(+)}(T, \widetilde{T}):=\frac{\left\langle\bar{T}^{\dot{\mu}} \mid \widetilde{\bar{T}}^{\dot{v}}\right\rangle_{\mathbb{J}}\left\langle\bar{T}_{\dot{\mu}} \mid \widetilde{\bar{T}}_{\dot{\nu}}\right\rangle_{\mathbb{J}}}{\left\langle\bar{T}^{\dot{\sigma}} \mid \bar{T}_{\dot{\sigma}}\right\rangle_{\mathbb{J}}\left\langle\widetilde{\bar{T}}^{\dot{\rho}} \mid \tilde{\bar{T}}_{\dot{\rho}}\right\rangle_{\mathbb{J}}},
\end{gathered}
$$

which are $\operatorname{OSp}(\mathcal{N} \mid 4 ; \mathbb{R})$-invariant. They are also invariant under arbitrary equivalence transformations (3.24). As follows from (3.27), $\omega_{(-)}(T, \widetilde{T})$ depends on the chiral variables $x_{(-)}, z_{(-)}$and $\theta$ at each point $T$ and $\widetilde{T}$, whilst $\omega_{(+)}(T, \widetilde{T})$ depends on the antichiral variables $x_{(+)}, z_{(+)}$and $\bar{\theta}$. This means that $\omega_{(-)}(T, \widetilde{T})$ and $\omega_{(+)}(T, \widetilde{T})$ are distinct. The three two-point functions (3.23) and (3.30) coincide only if the Grassmann variables $\theta$ and $\bar{\theta}$ are switched off.

This non-uniqueness is not surprising, since correlation functions could depend on the type of supersymmetric mul- tiplets and shortening conditions of local operators. ${ }^{7}$ The chirality condition is the simplest half-BPS constraint. With extended supersymmetry, other constraints can be imposed. The detailed classification goes beyond the scope of the present paper, though we expect the next two sections to provide techniques required to tackle such an analysis.

\section{Bi-supertwistor construction for $\operatorname{AdS}^{4 \mid 4 \mathcal{N}}$}

Along with the supertwistor realisation of compactified $\mathcal{N}$-extended Minkowski superspaces in four dimensions, $\overline{\mathbb{M}}^{4 \mid 4 \mathcal{N}}$, there also exists the so-called bi-supertwistor realisation for the same superspace which was introduced by Siegel $[52,53]$ (see [18] for a modern description). Here we describe its extension to $\operatorname{AdS}^{4 / 4 \mathcal{N}}$.

It should be mentioned that the bi-supertwistor construction of $\overline{\mathbb{M}}^{4 \mid 4 \mathcal{N}}$ was called "superembedding formalism" in [54-56]. Indeed, this construction may be viewed as a specific example of a general (super)embedding approach reviewed in [57] in application to superbranes. This construction was advocated in $[54-56,58,59]$ as a powerful alternative technique to compute correlation functions in conformal field theories, which is in a sense complementary to the more traditional superspace approaches pursued in [60-63].

Given a point in $\mathfrak{F}_{\mathcal{N}}$, we associate with it the graded antisymmetric matrices

$X_{A B}:=-2 \frac{T_{A}^{\mu} T_{B \mu}}{\left\langle T^{v} \mid T_{\nu}\right\rangle_{\mathbb{J}}}=-(-1)^{\varepsilon_{A} \varepsilon_{B}} X_{B A}$,

$\bar{X}_{A B}:=-2 \frac{\bar{T}_{A}^{\dot{\mu}} \bar{T}_{B \dot{\mu}}}{\left\langle\bar{T}^{\dot{v}} \mid \bar{T}_{\dot{v}}\right\rangle_{\mathbb{J}}}=-(-1)^{\varepsilon_{A} \varepsilon_{B}} \bar{X}_{B A}$.

These supermatrices are invariant under arbitrary equivalence transformations

$T^{\mu} \rightarrow T^{\nu} R_{\nu}^{\mu}, \quad R \in \mathrm{GL}(2, \mathbb{C})$,

\footnotetext{
${ }^{7}$ Here it is instructive to recall the following well-known results in $\mathcal{N}$-extended Minkowski superspace $\mathbb{M}^{4 \mid 4 \mathcal{N}}$ parametrised by Cartesian coordinates $z^{A}=\left(x^{a}, \theta_{i}^{\alpha}, \bar{\theta}_{\dot{\alpha}}^{i}\right)$, where $i=1, \ldots, \mathcal{N}$. This superspace possesses a one-parameter family of supersymmetric vector twopoint functions [50,51], $\zeta_{(\lambda)}^{a}\left(z, z^{\prime}\right)=\left(x-x^{\prime}\right)^{a}-\mathrm{i}\left(\theta-\theta^{\prime}\right){ }_{i} \sigma^{a} \bar{\theta}^{\prime i}+$ $\mathrm{i} \theta_{i}^{\prime} \sigma^{a}\left(\bar{\theta}-\bar{\theta}^{\prime}\right)^{i}-\lambda \mathrm{i}\left(\theta-\theta^{\prime}\right)_{i} \sigma^{a}\left(\bar{\theta}-\bar{\theta}^{\prime}\right)^{i}$, where $\lambda$ is a parameter. For any two values of the parameter, $\lambda_{1}$ and $\lambda_{2}$, the two-point function $\eta_{a b} \zeta_{\left(\lambda_{1}\right)}^{a}\left(z, z^{\prime}\right) \zeta_{\left(\lambda_{2}\right)}^{b}\left(z, z^{\prime}\right)$ is super-Poincaré invariant, with $\eta_{a b}$ being the Minkowski metric. We note that $\zeta_{(0)}^{a}\left(z, z^{\prime}\right)$ is real, whilst $\zeta_{(1)}^{a}\left(z, z^{\prime}\right)$ is antichiral with respect to $z$ and chiral with respect $z^{\prime}$.
} 
and therefore they may be used to parametrise $\operatorname{AdS}^{4 \mid 4 \mathcal{N}}$. The bi-supertwistors (4.1) have the following properties:

$$
\begin{aligned}
X_{[A B} X_{C D\}} & =0, \\
(-1)^{\varepsilon_{B}} X_{A B} \rrbracket^{B C} X_{C D} & =X_{A D}, \\
\mathbb{J}^{B A} X_{A B} & =2, \\
(-1)^{\varepsilon_{B}} X_{A B} \rrbracket^{B C} \bar{X}_{C D} & =0 .
\end{aligned}
$$

Making use of the results of [18], the bi-supertwistor formulation for $\operatorname{AdS}^{4 \mid 4 \mathcal{N}}$ defined by (4.3) may be shown to be equivalent to the supertwistor one described in Sect. 3.

\section{Harmonic/projective AdS superspaces}

The supertwistor realisations of $\operatorname{AdS}^{(3 \mid p, q)}$ and $\operatorname{AdS}^{4 \mid 4 \mathcal{N}}$, which have been described in Sects. 2 and 3 , make use of even supertwistors. In order to formulate AdS analogues of the harmonic $[64,65]$ and projective [66-68] superspaces, odd supertwistors must be taken into account. The corresponding technical details are analogous to the $3 \mathrm{D}$ and $4 \mathrm{D}$ flat-superspace constructions of Refs. [12,17] which built on earlier works $[13,14,16]$. This is why we provide such AdS formulations only in three dimensions.

Here we consider particular members of the family of 3D $(p, q)$ AdS superspaces, specifically $\operatorname{AdS}^{(3 \mid \mathcal{N}, 0)} \equiv$ $\operatorname{AdS}^{3 \mid 2 \mathcal{N}}$. For a fixed $\mathcal{N}=p+q$, the specific feature of $\operatorname{AdS}^{(3 \mid \mathcal{N}, 0)}$ and $\operatorname{Ad} S^{(3 \mid 0, \mathcal{N})}$ is that the corresponding $R$-symmetry subgroup of the isometry group (2.2) is maximal and coincides with the $R$-symmetry subgroup of the $\mathcal{N}$-extended superconformal group $\operatorname{OSp}(\mathcal{N} \mid 4 ; \mathbb{R})$, which is $\operatorname{SO}(\mathcal{N}){ }^{8}$ Superspace $\operatorname{AdS}^{3 / 2 \mathcal{N}}$ can be extended to $\operatorname{AdS}^{3 \mid 2 \mathcal{N}} \times \mathbb{X}_{1}^{\mathcal{N}}$, where the internal space $\mathbb{X}_{1}^{\mathcal{N}}$ is realised in terms of left complex odd supertwistors 9

$\Sigma_{\mathrm{L}}=\left(\begin{array}{c}\rho_{\bar{\alpha}} \\ \zeta_{\bar{I}}\end{array}\right), \quad \zeta_{\bar{I}} \neq 0$,

which are subject to the constraints

$\mathcal{P}_{\mathrm{L}}^{\mathrm{sT}} \mathbb{J}_{\mathrm{L}} \Sigma_{\mathrm{L}}=0, \quad \Sigma_{\mathrm{L}}^{\mathrm{sT}} \mathbb{J}_{\mathrm{L}} \Sigma_{\mathrm{L}}=0$,

and are defined modulo the equivalence relation

$\Sigma_{\mathrm{L}} \sim c \Sigma_{\mathrm{L}}, \quad c \in \mathbb{C} \backslash\{0\}$.

\footnotetext{
$\overline{8}$ The superspaces $\operatorname{AdS}^{(3 \mid \mathcal{N}, 0)}$ and $\operatorname{AdS}^{(3 \mid 0, \mathcal{N})}$ are related to each other by a parity transformation.

${ }^{9}$ One can also consider superspaces $\operatorname{AdS}^{3 \mid 2 \mathcal{N}} \times \mathbb{X}_{m}^{\mathcal{N}}$, for any integer $m \leq[\mathcal{N} / 2]$, with $[\mathcal{N} / 2]$ being the integer part of $\mathcal{N} / 2$. Space $\mathbb{X}_{m}^{\mathcal{N}}$ is realised in terms of $m$ left odd complex supertwistors $\Sigma^{i}$, with $i=$ $1, \ldots, m$, such that (i) the bodies of $\Sigma^{i}$ are linearly independent; (ii) the $\Sigma^{i}$ obey the constraints $\mathcal{P}_{\mathrm{L}}^{\mathrm{sT}} \mathbb{J}_{\mathrm{L}} \Sigma_{\mathrm{L}}^{i}=0$ and $\Sigma_{\mathrm{L}}^{i \mathrm{sT}} \mathbb{J}_{\mathrm{L}} \Sigma_{\mathrm{L}}^{j}=0$; and (iii) the $\Sigma^{i}$ are defined modulo the equivalence relation $\Sigma^{i} \sim \Sigma^{j} D_{j}{ }^{i}$, with $D \in \mathrm{GL}(m, \mathbb{C})$.
}

In the gauge (2.25), the above constraints take the form:

$\rho_{\bar{\alpha}}=\zeta_{\bar{I}} \vartheta_{\bar{I}}^{\bar{\beta}} \varepsilon_{\bar{\beta} \bar{\alpha}}, \quad \zeta_{\bar{I}} \zeta_{\bar{I}}=\mathrm{i} \rho_{\bar{\alpha}} \varepsilon^{\bar{\alpha} \bar{\beta}} \rho_{\bar{\beta}}$.

For $\mathcal{N}>2$ the internal manifold $\mathbb{X}_{1}^{\mathcal{N}}$ proves to be a symmetric space,

$\mathbb{X}_{1}^{\mathcal{N}}=\frac{\mathrm{SO}(\mathcal{N})}{\mathrm{SO}(\mathcal{N}-2) \times \mathrm{SO}(2)}, \quad \mathcal{N}>2$.

In the $\mathcal{N}=3$ case, the internal space $\mathbb{X}_{1}^{3}$ is $\mathbb{C} P^{1}$, while for $\mathcal{N}=4$ one obtains $\mathbb{X}_{1}^{4}=\mathbb{C} P^{1} \times \mathbb{C} P^{1}$, see [12] for the details.

It is obvious that the above construction naturally extends to the case of $(p, q)$ AdS superspaces with $p \geq q>0$. Technical details will be skipped.

\section{Conclusion}

In this paper we have presented supersymmetric extensions of the twistor descriptions of $\mathrm{AdS}_{3}$ and $\mathrm{AdS}_{4}$. Specifically, we have proposed supertwistor realisations of $(p, q)$ AdS superspaces in three dimensions and $\mathcal{N}$-extended AdS superspaces in four dimensions. In the three-dimensional case, we have also presented harmonic/projective superspace formulations of $(p, q)$ AdS supersymmetry, and these results can be readily extended to four dimensions.

One of the main results of our paper is the construction of manifestly supersymmetric two-point functions in $\operatorname{AdS}^{(3 \mid p, q)}$ and $\mathrm{AdS}^{4 \mid 4 \mathcal{N}}$. In Minkowski backgrounds, the embedding approach is known to be a powerful framework for deciphering the structure of correlation functions in conformal field theories - see, e.g., [54-56,58,59,69]. Analogously, it is of interest for several applications to study $n$-point correlation functions in AdS by employing symmetry arguments, see, e.g., [49] and references therein for a recent discussion in the non-supersymmetric case. The results of our work open new avenues to perform manifestly supersymmetric studies of correlation functions in $\mathrm{AdS}_{3}$ and $\mathrm{AdS}_{4}$. We aim to look into this direction in the near future.

Acknowledgements We are grateful to Michael Ponds for comments on the manuscript. The work of SMK is supported in part by the Australian Research Council, project No. DP200101944. The work of GT-M is supported by the Australian Research Council (ARC) Future Fellowship FT180100353, and by the Capacity Building Package of the University of Queensland.

Data Availability Statement This manuscript has no associated data or the data will not be deposited. [Authors' comment: The theoretical research in this manuscript is pen and paper and does not require data management.]

Open Access This article is licensed under a Creative Commons Attribution 4.0 International License, which permits use, sharing, adaptation, distribution and reproduction in any medium or format, as long as you 
give appropriate credit to the original author(s) and the source, provide a link to the Creative Commons licence, and indicate if changes were made. The images or other third party material in this article are included in the article's Creative Commons licence, unless indicated otherwise in a credit line to the material. If material is not included in the article's Creative Commons licence and your intended use is not permitted by statutory regulation or exceeds the permitted use, you will need to obtain permission directly from the copyright holder. To view a copy of this licence, visit http://creativecomm ons.org/licenses/by/4.0/.

Funded by SCOAP ${ }^{3}$.

\section{A Compactified $(p, q)$ Minkowski superspace in two dimensions}

For completeness, in this appendix we describe a supertwistor realisation of 2D compactified Minkowski superspace $\overline{\mathbb{M}}^{(2 \mid p, q)}$. This superspace will be identified with

$\overline{\mathbb{M}}^{(2 \mid p, q)}=\Lambda_{(p, q)} / \sim$

Here $\Lambda_{(p, q)}$ is the space of real even supertwistor pairs $\left(T_{\mathrm{L}}, T_{\mathrm{R}}\right)$, where $T_{\mathrm{L}}$ and $T_{\mathrm{R}}$ are left and right even real supertwistors of the form (2.3) and (2.4), respectively, with nonzero bosonic parts,

$\mathfrak{T}_{\mathrm{L}}:=\left(T_{\bar{\alpha}}\right) \neq 0, \quad \mathfrak{T}_{\mathrm{R}}:=\left(T_{\underline{\alpha}}\right) \neq 0$.

The equivalence relation in (A.1) is defined by

$\left(T_{\mathrm{L}}, T_{\mathrm{R}}\right) \sim\left(\rho_{\mathrm{L}} T_{\mathrm{L}}, \rho_{\mathrm{R}} T_{\mathrm{R}}\right), \quad \rho_{\mathrm{L}}, \rho_{\mathrm{R}} \in \mathbb{R}-\{0\}$.

The supergroup (2.2) acts on $\Lambda_{(p, q)}$ by the rule

$$
\begin{aligned}
& \left(g_{\mathrm{L}}, g_{\mathrm{R}}\right)\left(T_{\mathrm{L}}, T_{\mathrm{R}}\right):=\left(g_{\mathrm{L}} T_{\mathrm{L}}, g_{\mathrm{R}} T_{\mathrm{R}}\right), \\
& \left(g_{\mathrm{L}}, g_{\mathrm{R}}\right) \in \operatorname{OSp}(p \mid 2 ; \mathbb{R}) \times \operatorname{OSp}(q \mid 2 ; \mathbb{R}) .
\end{aligned}
$$

This action is naturally extended to the quotient space $\Lambda_{(p, q)} / \sim$. The latter proves to be a homogeneous space of $\operatorname{OSp}(p \mid 2 ; \mathbb{R}) \times \operatorname{OSp}(q \mid 2 ; \mathbb{R})$.

Let us define one-forms

$\omega_{\mathrm{L}}=-T_{\mathrm{L}}^{\mathrm{sT}} \mathbb{J}_{\mathrm{L}} \mathrm{d} T_{\mathrm{L}}, \quad \omega_{\mathrm{R}}=-T_{\mathrm{R}}^{\mathrm{sT}} \mathbb{J}_{\mathrm{R}} \mathrm{d} T_{\mathrm{R}}$.

They have the following properties: (i) $\omega_{\mathrm{L}}$ and $\omega_{\mathrm{R}}$ are invariant under the action of $\operatorname{OSp}(p \mid 2 ; \mathbb{R}) \times \operatorname{OSp}(q \mid 2 ; \mathbb{R}) ;$ and (ii) $\omega_{\mathrm{L}}$ and $\omega_{\mathrm{R}}$ scale under point-dependent (local) equivalence transformations,

$\omega_{\mathrm{L}} \rightarrow \rho_{\mathrm{L}}^{2} \omega_{\mathrm{L}}, \quad \omega_{\mathrm{R}} \rightarrow \rho_{\mathrm{R}}^{2} \omega_{\mathrm{R}}$.

Therefore we can define a superconformal metric on $\overline{\mathbb{M}}^{(2 \mid p, q)}$ by the rule

$\mathrm{d} s^{2}=\omega_{\mathrm{L}} \omega_{\mathrm{R}}$.

In order to get a better feeling for the above construction, let us consider the non-supersymmetric case, $p=q=0$. The elements of $\Lambda=\Lambda_{(0,0)}$ are all possible pairs $\left(T_{\mathrm{L}}, T_{\mathrm{R}}\right)=$ $\left(T_{\bar{\alpha}}, T_{\underline{\alpha}}\right)$, where the real two-component spinors $T_{\bar{\alpha}}$ and $T_{\underline{\alpha}}$ are non-zero. The freedom to perform equivalence transformations (A.3) can be partially fixed by imposing the conditions

$\left(T_{\overline{1}}\right)^{2}+\left(T_{\overline{2}}\right)^{2}=1, \quad\left(T_{\underline{1}}\right)^{2}+\left(T_{\underline{2}}\right)^{2}=1$.

In this gauge, the equivalence relation (A.3) reduces to $T_{\bar{\alpha}} \sim$ $-T_{\bar{\alpha}}$ and $T_{\alpha} \sim-T_{\alpha}$. It is seen that the quotient space $\Lambda / \sim$ is $S^{1} \times S^{1}$.

Instead of imposing the conditions (A.8), we can introduce inhomogeneous (North-chart) coordinates for the onespheres,

$T_{\mathrm{L}}=\left(\begin{array}{c}x_{\mathrm{L}} \\ 1\end{array}\right), \quad T_{\mathrm{R}}=\left(\begin{array}{c}x_{\mathrm{R}} \\ 1\end{array}\right)$.

Then the one-forms (A.6) take the form

$\omega_{\mathrm{L}}=\mathrm{d} x_{\mathrm{L}}, \quad \omega_{\mathrm{R}}=\mathrm{d} x_{\mathrm{R}}$,

and the metric (A.7) becomes $\mathrm{d} s^{2}=\mathrm{d} x_{\mathrm{L}} \mathrm{d} x_{\mathrm{R}}$. Given a group element

$g_{\mathrm{L}}=\left(\begin{array}{ll}a & b \\ c & d\end{array}\right) \in G_{\mathrm{L}}=\mathrm{Sp}(2, \mathbb{R}) \cong \mathrm{SL}(2, \mathbb{R})$,

it acts on $T_{\mathrm{L}}$, eq. (A.9), by the fractional linear transformation

$x_{\mathrm{L}} \rightarrow \frac{a x_{\mathrm{L}}+b}{c x_{\mathrm{L}}+d} \Longrightarrow \mathrm{d} x_{\mathrm{L}} \rightarrow \frac{\mathrm{d} x_{\mathrm{L}}}{\left(c x_{\mathrm{L}}+d\right)^{2}}$.

Given a group element $g_{\mathrm{R}} \in G_{\mathrm{R}}=\operatorname{Sp}(2, \mathbb{R})$, it generates a similar fractional linear transformation of $x_{\mathrm{R}}$. Under the action of $\left(g_{\mathrm{L}}, g_{\mathrm{R}}\right) \in G_{\mathrm{L}} \times G_{\mathrm{R}}$, the metric $\mathrm{d} s^{2}=\mathrm{d} x_{\mathrm{L}} \mathrm{d} x_{\mathrm{R}}$ scales.

\section{References}

1. S.J. Avis, C.J. Isham, D. Storey, Quantum field theory in anti-de Sitter space-time. Phys. Rev. D 18, 3565 (1978)

2. C.P. Burgess, C.A. Lütken, Propagators and effective potentials in anti-de Sitter space. Phys. Lett. B 153, 137-141 (1985)

3. C.J.C. Burges, D.Z. Freedman, S. Davis, G.W. Gibbons, Supersymmetry in anti-de Sitter space. Ann. Phys. 167, 285 (1986)

4. B. Allen, T. Jacobson, Vector two-point functions in maximally symmetric spaces. Commun. Math. Phys. 103, 669 (1986)

5. B. Allen, C.A. Lütken, Spinor two-point functions in maximally symmetric spaces. Commun. Math. Phys. 106, 201 (1986). https:// doi.org/10.1007/BF01454972

6. E. D'Hoker, D.Z. Freedman, S.D. Mathur, A. Matusis, L. Rastelli, Graviton and gauge boson propagators in $\operatorname{AdS}(d+1)$. Nucl. Phys. B 562, 330 (1999). arXiv:hep-th/9902042 [hep-th]

7. A. Ferber, Supertwistors and conformal supersymmetry. Nucl. Phys. B 132, 55 (1978)

8. Yu.I. Manin, Holomorphic supergeometry and Yang-Mills superfields. J. Sov. Math. 30, 1927 (1985)

9. Yu.I. Manin, Gauge Field Theory and Complex Geometry, Springer, Berlin (1988)

10. M. Kotrla, J. Niederle, Supertwistors and superspace. Czech. J. Phys. B 35, 602 (1985) 
11. P.S. Howe, M.I. Leeming, Harmonic superspaces in low dimensions. Class. Quantum Gravity 11, 2843 (1994). arXiv:hep-th/9408062

12. S.M. Kuzenko, J.-H. Park, G. Tartaglino-Mazzucchelli, R. Unge, Off-shell superconformal nonlinear sigma-models in three dimensions. JHEP 1101, 146 (2011). arXiv:1011.5727 [hep-th]

13. A.A. Rosly, Gauge fields in superspace and twistors. Class. Quantum Gravity 2, 693 (1985)

14. J. Lukierski, A. Nowicki, General superspaces from supertwistors. Phys. Lett. B 211, 276 (1988)

15. G.G. Hartwell, P.S. Howe, $(\mathrm{N}, \mathrm{p}, \mathrm{q})$ harmonic superspace. Int. J. Mod. Phys. A 10, 3901-3920 (1995). arXiv:hep-th/9412147

16. P.S. Howe, G.G. Hartwell, A superspace survey. Class. Quantum Gravity 12, 1823 (1995)

17. S.M. Kuzenko, On compactified harmonic/projective superspace, 5D superconformal theories, and all that. Nucl. Phys. B 745, 176 (2006). arXiv:hep-th/0601177

18. S.M. Kuzenko, Conformally compactified Minkowski superspaces revisited. JHEP 1210, 135 (2012). arXiv: 1206.3940 [hep-th]

19. E.I. Buchbinder, S.M. Kuzenko, I.B. Samsonov, Superconformal field theory in three dimensions: correlation functions of conserved currents. JHEP 06, 138 (2015). arXiv:1503.04961 [hep-th]

20. S.M. Kuzenko, D. Sorokin, Superconformal structures on the threesphere. JHEP 10, 080 (2014). arXiv:1406.7090 [hep-th]

21. P.S. Howe, U. Lindström, Local supertwistors and conformal supergravity in six dimensions. Proc. R. Soc. Lond. A 476(2243), 20200683 (2020). arXiv:2008.10302 [hep-th]

22. P.S. Howe, U. Lindström, Superconformal geometries and local twistors. JHEP 04, 140 (2021). arXiv:2012.03282 [hep-th]

23. P. Claus, M. Gunaydin, R. Kallosh, J. Rahmfeld, Y. Zunger, Supertwistors as quarks of $S U(2,2 \mid 4)$. JHEP 05, 019 (1999). arXiv:hep-th/9905112

24. P. Claus, J. Rahmfeld, Y. Zunger, A simple particle action from a twistor parametrization of $\operatorname{AdS}(5)$. Phys. Lett. B 466, 181-189 (1999). arXiv:hep-th/9906118

25. P. Claus, R. Kallosh, J. Rahmfeld, BRST quantization of a particle in AdS(5). Phys. Lett. B 462, 285-293 (1999). arXiv:hep-th/9906195

26. I.A. Bandos, J. Lukierski, C. Preitschopf, D.P. Sorokin, OSp supergroup manifolds, superparticles and supertwistors. Phys. Rev. D 61, 065009 (2000). arXiv:hep-th/9907113

27. Y. Zunger, Twistors and actions on coset manifolds. Phys. Rev. D 62, 024030 (2000). arXiv:hep-th/0001072

28. M. Cederwall, Geometric construction of AdS twistors. Phys. Lett. B 483, 257-263 (2000). arXiv:hep-th/0002216

29. M. Cederwall, AdS twistors for higher spin theory. AIP Conf. Proc. 767(1), 96-105 (2005). arXiv:hep-th/0412222

30. A.S. Arvanitakis, A.E. Barns-Graham, P.K. Townsend, Anti-de Sitter particles and manifest (super)isometries. Phys. Rev. Lett. 118(14), 141601 (2017). arXiv:1608.04380 [hep-th]

31. A.S. Arvanitakis, A.E. Barns-Graham, P.K. Townsend, Twistor description of spinning particles in AdS. JHEP 01, 059 (2018). arXiv: 1710.09557 [hep-th]

32. D.V. Uvarov, Supertwistor formulation for massless superparticle in $A d S_{5} \times S^{5}$ superspace. Nucl. Phys. B 936, 690-713 (2018). arXiv: 1807.08318 [hep-th]

33. R.R. Metsaev, A.A. Tseytlin, Type IIB superstring action in AdS(5) x S**5 background. Nucl. Phys. B 533, 109-126 (1998). arXiv:hep-th/9805028

34. S.M. Kuzenko, U. Lindström, G. Tartaglino-Mazzucchelli, Threedimensional (p, q) AdS superspaces and matter couplings. JHEP 1208, 024 (2012). arXiv:1205.4622 [hep-th]

35. P.S. Howe, J.M. Izquierdo, G. Papadopoulos, P.K. Townsend, New supergravities with central charges and Killing spinors in $2+1$ dimensions. Nucl. Phys. B 467, 183 (1996). arXiv:hep-th/9505032
36. S.M. Kuzenko, U. Lindström, G. Tartaglino-Mazzucchelli, Offshell supergravity-matter couplings in three dimensions. JHEP 1103, 120 (2011). arXiv:1101.4013 [hep-th]

37. A. Achúcarro, P.K. Townsend, A Chern-Simons action for threedimensional anti-de Sitter supergravity theories. Phys. Lett. B 180, 89 (1986)

38. I.A. Bandos, E. Ivanov, J. Lukierski, D. Sorokin, On the superconformal flatness of AdS superspaces. JHEP 06, 040 (2002). arXiv:hep-th/0205104

39. S.J. Gates Jr., M.T. Grisaru, M. Roček, W. Siegel, Superspace, or one thousand and one lessons in supersymmetry. Front. Phys. 58, 1 (1983). arXiv:hep-th/0108200

40. S.M. Kuzenko, G. Tartaglino-Mazzucchelli, Three-dimensional $\mathrm{N}=2$ (AdS) supergravity and associated supercurrents. JHEP 1112, 052 (2011). arXiv:1109.0496 [hep-th]

41. I.L. Buchbinder, S.M. Kuzenko, Ideas and Methods of Supersymmetry and Supergravity or a Walk Through Superspace. IOP, Bristol (1995) (Revised Edition: 1998)

42. B.W. Keck, An alternative class of supersymmetries. J. Phys. A 8, 1819 (1975)

43. B. Zumino, Nonlinear realization of supersymmetry in de Sitter space. Nucl. Phys. B 127, 189 (1977)

44. E.A. Ivanov, A.S. Sorin, Superfield formulation of $\operatorname{OSp}(1,4)$ supersymmetry. J. Phys. A 13, 1159 (1980)

45. S.M. Kuzenko, U. Lindström, M. Roček, G. TartaglinoMazzucchelli, 4D N = 2 supergravity and projective superspace. JHEP 0809, 051 (2008). arXiv:0805.4683

46. S.M. Kuzenko, G. Tartaglino-Mazzucchelli, Field theory in 4D $\mathrm{N}=2$ conformally flat superspace. JHEP 0810, 001 (2008). arXiv:0807.3368 [hep-th]

47. D. Butter, S.M. Kuzenko, $N=2$ AdS supergravity and supercurrents. JHEP 07, 081 (2011). arXiv:1104.2153 [hep-th]

48. D. Butter, S.M. Kuzenko, U. Lindström, G. TartaglinoMazzucchelli, Extended supersymmetric sigma models in $\mathrm{AdS}_{4}$ from projective superspace. JHEP 05, 138 (2012). arXiv:1203.5001 [hep-th]

49. D.J. Binder, D.Z. Freedman, S.S. Pufu, A bispinor formalism for spinning Witten diagrams. arXiv:2003.07448 [hep-th]

50. V.P. Akulov, D.V. Volkov, Goldstone fields with spin 1/2. Theor. Math. Phys. 18, 28 (1974)

51. V.P. Akulov, D.V. Volkov, Teor. Mat. Fiz. 18, 39 (1974)

52. W. Siegel, Green-Schwarz formulation of self-dual superstring. Phys. Rev. D 47, 2512 (1993). arXiv:hep-th/9210008

53. W. Siegel, Super multi-instantons in conformal chiral superspace. Phys. Rev. D 52, 1042 (1995). arXiv:hep-th/9412011

54. W.D. Goldberger, W. Skiba, M. Son, Superembedding methods for 4d N=1 SCFTs. Phys. Rev. D 86, 025019 (2012). arXiv:1112.0325 [hep-th]

55. M. Maio, Superembedding methods for $4 \mathrm{~d}$ N-extended SCFTs. Nucl. Phys. B 864, 141 (2012). arXiv:1205.0389 [hep-th]

56. W.D. Goldberger, Z.U. Khandker, D. Li, W. Skiba, Superembedding methods for current superfields. Phys. Rev. D 88, 125010 (2013). arXiv:1211.3713 [hep-th]

57. D.P. Sorokin, Superbranes and superembeddings. Phys. Rep. 329, 1 (2000). arXiv:hep-th/9906142

58. A.L. Fitzpatrick, J. Kaplan, Z.U. Khandker, D. Li, D. Poland, D. Simmons-Duffin, Covariant approaches to superconformal blocks. JHEP 08, 129 (2014). arXiv:1402.1167 [hep-th]

59. Z.U. Khandker, D. Li, D. Poland, D. Simmons-Duffin, $\mathcal{N}=1$ superconformal blocks for general scalar operators. JHEP 08, 049 (2014). arXiv:1404.5300 [hep-th]

60. H. Osborn, $N=1$ superconformal symmetry in fourdimensional quantum field theory. Ann. Phys. 272, 243 (1999). arXiv:hep-th/9808041

61. J.-H. Park, Superconformal symmetry and correlation functions. Nucl. Phys. B 559, 455 (1999). arXiv:hep-th/9903230 
62. S.M. Kuzenko, S. Theisen, Correlation functions of conserved currents in $\mathrm{N}=2$ superconformal theory. Class. Quantum Gravity 17, 665 (2000). arXiv:hep-th/9907107

63. J.-H. Park, Superconformal symmetry in three-dimensions. J. Math. Phys. 41, 7129 (2000). arXiv:hep-th/9910199

64. A. Galperin, E. Ivanov, S. Kalitsyn, V. Ogievetsky, E. Sokatchev, Unconstrained $\mathrm{N}=2$ matter, Yang-Mills and supergravity theories in harmonic superspace. Class. Quantum Gravity 1, 469 (1984)

65. A.S. Galperin, E.A. Ivanov, V.I. Ogievetsky, E.S. Sokatchev, Harmonic Superspace (Cambridge University Press, Cambridge, 2001)
66. A. Karlhede, U. Lindström, M. Roček, Self-interacting tensor multiplets in $\mathrm{N}=2$ superspace. Phys. Lett. B 147, 297 (1984)

67. U. Lindström, M. Roček, New hyperkähler metrics and new supermultiplets. Commun. Math. Phys. 115, 21 (1988)

68. U. Lindström, M. Roček, $\mathrm{N}=2$ super Yang-Mills theory in projective superspace. Commun. Math. Phys. 128, 191 (1990)

69. M.S. Costa, J. Penedones, D. Poland, S. Rychkov, Spinning conformal correlators. JHEP 11, 071 (2011). arXiv:1107.3554 [hep-th] 